\title{
Um Sistema Especialista Probabilístico para o Apoio a Análise de Planos de Negócios de Empresas de Base Tecnológica
}

\author{
Jordan Pauleski Juliani', Luis Henrique Bogo ${ }^{2}$, Raphael Winckler de Bettio ${ }^{3}$, Alejandro \\ Martins Rodrigues ${ }^{4}$, Heleno Fulber ${ }^{5}$ \\ 1,2,3,4,5 Programa de Pós-Graduação em Engenharia e Gestão do Conhecimento, Universidade Federal de \\ Santa Catarina, Trindade, Florianópolis, SC, Brasil \\ \{jordan, bogo, raphael, hfulber\}@egc.ufsc.br
}

\begin{abstract}
Resumo - Este artigo apresenta uma proposta de desenvolvimento de um sistema especialista probabilístico como forma de apoiar a análise dos planos de negócio de empresas de tecnologia, por parte dos consultores especializados, estruturados para fim de ingresso em incubadoras de empresas. Destacase neste contexto o trabalho intrinsicamente subjetivo e pouco metodológico de análise de planos de negócio, como forma de julgar novos projetos de empresas, gerando como resultado para as incubadoras um alto risco de não apoiar projetos com real potencial de desenvolvimento, desestimulando o empreendedorismo e prejudicando a economia local.
\end{abstract}

Palavras-chave: Sistemas Especialista Probabilísticos, Plano de Negócio, Inteligência Artificial.

\begin{abstract}
This article presents a proposal of specialist probabilist system development as form to support the analysis of technology companies business plans, on the part of the specialized, structuralized consultants for its ingression in firms that support it. This task is essentially a subjective work and there is little method applied on it, as form to judge new companies projects, generating as result a high risk on the projects investment.
\end{abstract}

Key-words: Probabilist Expert Systems, Bussiness Plan, Artificial Intelligence.

\section{Introdução}

As demandas por soluções tecnológicas provocadas pela era da informação, caracterizada pela alta conectividade, pelo grande volume de informações produzidas pelas corporações, e pela rápida evolução dos padrões e sistemas computacionais, provocam o aquecimento da indústria do software, como forma de atender as necessidades organizacionais de gestão através de recursos informáticos. Percebendo 0 crescimento deste arranjo produtivo, o governo brasileiro em parceria com empresas privadas incentivam o surgimento de empresas de base tecnológica, através da formação de incubadoras de empresas, que provem toda a infra-estrutura necessária para o funcionamento desses negócios, inclusive oferecendo serviços de consultoria com foco em apoiar e acompanhar a evolução dos empreendimentos.

O processo de incubação normalmente inicia-se por meio da estruturarão de um plano de negócios, formatado pelo empreendedor interessado, através do qual são mapeadas as principais variáveis do negócio, sistematizando assim suas idéias e planejando de forma mais eficiente o investimento. Finalizada esta etapa, o plano de negócios é encaminhado para uma banca avaliadora, composta de consultores especializados, que aprecia o projeto baseandose em suas experiências pessoais, nas informações produzidas pelo mercado, pela própria incubadora, e naquelas contidas no plano, deliberando sobre a continuidade do processo de incubação do empreendimento proposto.

Neste contexto concentra-se o esforço desta pesquisa, visto o alto nível de subjetividade no processo de análise e da falta de critérios quantitativos para o julgamento dos planos. Visando minimizar os erros na avaliação, propõese o desenvolvimento de um sistema especialista probabilístico, proveniente da disciplina de inteligência artificial, como forma de apoiar a análise dos planos de negócio por parte dos consultores especializados.

A partir do levantamento bibliográfico não foram encontrados trabalhos aplicando sistemas especialistas probabilísticos nesta área de definição, o que dá a este trabalho um ponto forte no quesito originalidade. Em outras áreas alguns trabalhos vêm sendo realizados. Entre os sistemas pesquisados destacamos a aplicação de sistemas especialistas probabilísticos para 0 apoio ao diagnóstico de potencial econômico dos 
municípios de Santa Catarina e a aplicação na avaliação e prevenção de evasão escolar [4].

\section{Planos de Negócios}

O plano de negócio é um documento que possibilita o entendimento completo, relacionado a uma área de negócios, de um determinado empreendimento, que passou a ter destaque com - aumento da importância das pequenas empresas na economia das nações e com o crescimento das atividades de incentivo ao empreendedorismo [6]. de negócios:

Salim (2001) apud [6] assim define plano

é um documento que contém a caracterização do negócio, sua forma de operar, suas estratégias, seu plano para conquistar uma fatia do mercado e as projeções de despesas, receitas e resultados financeiros.

Não existe uma estrutura rígida ou um modelo único de Plano de Negócio, mas é necessário que o mesmo tenha uma série de seções com finalidade específica, que obedeça a certos padrões e seja organizada com uma seqüência lógica para facilitar a compreensão do negócio, sua organização, objetivos, produtos e/ou serviços, mercado alvo, estratégias mercadológicas e situação financeira.

Algumas formas de uso dos planos de negócio [5]:

- permite realizar controle gerencial para acompanhamento, avaliação e controle das fases do projeto da empresas

- possibilita identificar riscos e propor ações para eliminá-los ou, pelo menos, minimizá-los;

- viabiliza o conhecimento do o mercado e a definição de estratégias

- descreve um negócio: os motivos da existência da oportunidade de negócio, como o empreendedor pretende utilizá-la e como buscar e gerenciar os recursos para aproveitá-la;

- representa um instrumento de comunicação eficiente entre os envolvidos na operação e de negociação interna e externa para administrar a interdependência com os sócios, empregados, financiadores, incubadoras, clientes, fornecedores, bancos, etc;

- permite analisar e avaliar o desempenho financeiro do empreendimento.

\section{Sistemas Especialistas Probabilísticos}

Sistemas especialistas são programas de computador projetados para modelar a habilidade de especialistas humanos de solucionar problemas, sendo as principais áreas de aplicação, as finanças, a indústria e a medicina [1]. Os principais tipos de problemas resolvidos pelos sistemas especialistas, dentre eles pode-se destacar: o controle, o projeto, a interpretação, o diagnóstico, o monitoramento, o planejamento, a seleção e a simulação [1].

Sistemas Especialistas são sistemas computacionais que resolvem problemas de forma similar aos especialistas humanos, tratando-se de um conhecimento profundo sobre campos específicos do conhecimento [7].

As principais características de um sistema especialista são:

1. habilidade para trabalhar ao nível do especialista;

2. representa o conhecimento específico da maneira que o especialista pensa;

3. incorpora o processo de explanação e formas de manipulação de incerteza e

4. é normalmente, pertinente a problemas que podem ser simbolicamente representados.

A arquitetura de Sistemas Especialistas está baseada em três elementos básicos: a memória de trabalho, a base de conhecimento e a máquina de inferência, como representado na figura 1.

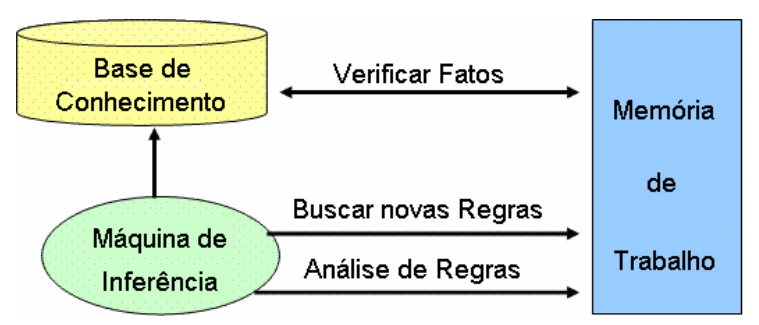

Figura 1 - Arquitetura dos Sistemas Especialistas

A base de conhecimento é representada pelo agrupamento de conhecimento representado mediante uma técnica adequada ao sistema em questão.

Memória de trabalho como sendo os fatos de um problema que são descobertos durante uma consulta [1]. Tais fatos podem ser informados pelo usuário do sistema ou fornecidos através da avaliação de uma das regras da base de conhecimento.

A máquina de inferência contém o conhecimento geral que direciona e controla o raciocínio do sistema. O mecanismo de inferência, executa tarefas da maior importância. Examina os fatos e regras existentes na base de dados, acrescentando novos fatos sempre que possível, na medida das interpretações e conclusões que executa. Basicamente ela se responsabiliza pela sequência de ações que deverão ser encadeadas, ou pela sequência de regras aplicadas.

Conforme destaca [3]: "a maneira mais amplamente utilizada para representar o conhecimento do domínio de aplicação nos sistemas especialistas é como um conjunto de 
regras SE - ENTÃO, que por vezes estão ligados a um sistema que define os objetos que ocorrem nas regras. Em alguns casos há dificuldades de adaptar o conhecimento para regras sendo necessária a utilização de técnicas como a do raciocínio probabilístico como uma das formas de representação do conhecimento incerto". Neste sentido, visando sanar a problemática de representar a incerteza surge o conceito dos sistemas especialistas probabilísticos, trabalhando com o conceito do Teorema de Bayes onde a partir dos dados de entrada são calculadas as relações existentes dentro do sistema. Neste contexto as incertezas do domínio são representadas em probabilidades, que definem a chance desta acontecer.

As redes bayesianas são empregadas nas bases de conhecimento definem as relações causais e valores probabilísticos.

Para a determinação do relacionamento das variáveis dentro do sistema as redes bayesianas utilizam-se dos seguintes cálculos [4]: $P(A)=P\left(A^{\prime}\right)$ onde:

$O$ cálculo da probabilidade do acontecimento de $A$, se dá calculando-se o valor de A'. O valor A' consiste no cálculo da probabilidade do acontecimento de $\mathrm{A}$ em todas as regras.

- $P(B / A)=\left(P(B)^{*} P(A / B) / P(A)\right)$ onde:

- $P(B / A)=$ corresponde a probabilidade de acontecer B, se A aconteceu.

- $P(B)=$ probabilidade do acontecimento de $B$; este valor é determinado pelo sistema.

- $P(A / B)=$ probabilidade de $A$ acontecer se $B$ for verdadeiro.

- $\mathrm{P}(\mathrm{A})=$ cálculo da probabilidade de $\mathrm{A}$ acontecer em todas as evidências.

\section{Descrição da Aplicação}

O processo de engenharia de conhecimento para a estruturação do sistema especialista para o apoio ao processo de análise de planos de negócios de empresas de base tecnológica iniciou-se através da identificação dos atributos e respectivos valores, utilizados pelos especialistas durante a análise dos planos, tendo como base a estrutura de um plano de negócios utilizados por empresas de base tecnológica.

Através de entrevistas semi-estruturadas com consultores especializados no julgamento de planos de negócio, foram estruturadas as regras de produção de codificam o conhecimento empregado no processo de análise, bem como suas probabilidades.

Como resultado os seguintes atributos foram identificados e agrupados em 5 tópicos, conforme apresentado no quando abaixo:

\section{O NEGÓCIO}

1.1. Missão e foco

Adequada

Pouco adequada

Inadequado

1.2. Perfil dos empreendedores

Qualificados

Pouco qualificados

Não qualificados

1.3. Produto ou serviço - nível de inovação Alto

Médio

Baixo

Nenhum

1.4. Produto ou serviço - demanda

Alta

Média

Baixa

Nenhuma

1.5. Produto ou serviço - Tecnologia incorporada

Vanguarda - com capacidade de evolução Vanguarda - sem capacidade de evolução Obsoleta

1.6. Produto ou serviço - Nível de rentabilidade

Alta

Média

Baixa

2. PLANEJAMENTO DE OPERACIONAL

2.1. Estrutura organizacional

Por função

Por departamento

2.2. Sistema de produção - processos

Adequada

Pouco adequada

Inadequado

2.3. Sistema de produção - dimensionamento Adequada

Pouco adequada

Inadequado

2.4. Fornecedores

Adequados

Pouco adequados

Inadequados

2.5. Parceiros

Adequados

Pouco adequados

Inadequados

\section{PLANEJAMENTO DE MARKETING}

3.1. Situação de mercado

Favorável

Pouco Favorável

Desfavorável

3.2. Tipo de concorrência predominante Direta

Indireta 
3.3. Nível de concorrência

Alta

Média

Baixa

Nenhuma

3.4. Localização

Adequada

Pouco adequada

Inadequado

3.5. Promoção

Adequada

Pouco adequada

Inadequado

3.6. Política de preços

Adequada

Pouco adequada

Inadequado

3.7. Canais de distribuição

Adequada

Pouco adequada

Inadequado

3.8. Publicidade e propaganda

Adequada

Pouco adequada

Inadequado

\section{PLANEJAMENTO FINANCEIRO}

4.1. Capital de giro

Adequada

4.2. Alcance de ponto de equilíbrio

Curto prazo

Médio prazo

Longo prazo

4.3. Estrutura de custos

Super dimensionado

Bem dimensionado

Sub dimensionado

\subsection{Projeção de vendas}

Super dimensionada

Bem dimensionada

Sub dimensionada

\section{RESULTADO DA ANÁLISE}

\subsection{Sucesso do empreendimento}

Muito provável

Bem provável

Pouco provável

Improvável

Para fins de avaliação do modelo proposto para o sistema as seguintes regras de produção foram geradas:

* ((Missao_Foco=Adequada) AND

(Perfil_Empreendores=Qualificados) AND

(Produto_Inovacao=Medio) AND

(Produto_Demanda=Media) AND

(Produto_Tecnologia=Vanguarda_Com_Capa

cidade Evolucao)) |

(Sucesso_Empreendimento=Bem_Provavel)
* ((Tipo Concorrencia=Direta) AND

(Nivel_Concorrencia=Alta) AND

(Politica_Precos=Similar_Ao_Mercado) AND

(Promocao=Adequada) AND

(Canais_Distribuicao=Pouco_Adequada) AND

(Publicidade_Propaganda=Āequada)) |

(Sucesso Empreendimento=Bem Provavel)

* ((Projecao_Vendas=Sub_Dimensionada) AND

(Estrutura_Organizacional=Pouco_Adequada)

AND

(Sistema_Producao_Processos=Pouco_Adeq uada)) |

(Sucesso_Empreendimento=Pouco_Provavel)

- ((Capital_Giro=Necessario) AND

(Situacao Mercado=Favoravel) AND

(Alcance_Ponto_Equilibrio=Medio_Prazo)

AND

(Estrutura Custos=Super Dimensionada)

AND (Projecao_Vendas=Bem_Dimensionada)

AND (Nivel Rentabilidade=Medio)) |

(Sucesso_Empreendimento=Muito_Provavel)

- ((Fornecedores=Pouco_Adequados) AND

(Parceiros=Pouco_Adequados)) |

((Sistema_Producāo_Processos=Pouco_Adeq uada) AND

(Sistema_Producao_Dimensionamento=Pouc o_Adequado))

* ((Produto Inovacao=Baixo) AND

(Missao_Foco=Pouco_Adequada)) |

(Sucesso_Empreendimento=Improvavel)

* ((Nivel_Concorrencia=Media) AND

(Produto Inovacao=Baixo)) |

(Sucesso_Empreendimento=Improvavel)

* ((Estrutura Custos=Bem Dimensionada) AND

(Nivel_Rentabilidade=Baixo))

(Sucesso Empreendimento=Pouco Provavel)

* ((Produto_Demanda=Media) AND

(Canais Distribuicao=Pouco Adequada) AND

(Publicidade_Propaganda=Adequada))

(Sucesso Empreendimento=Bem Provavel)

- (Missao_Foco=Pouco_Adequada)

(Sucesso_Empreendimento=Improvavel)

Utilizando-se da Shell SPIRIT ${ }^{1}$, o sistema especialista probabilístico foi codificado, a partir da definição dos atributos e valores, e suas relações, expressas através das regras de produção.

O shell disponibiliza uma interface gráfica para a implementação da aplicação, possibilitando a criação de variáveis de diversos tipos e utilizando os conceitos de redes baeysianas, incorporados nas regras de produção.

As regras e as variáveis são associadas às probabilidades, através das quais a rede realiza o seu aprendizado, permitindo com que a inferência possa ser realizada sobre a base para a seja realizado o diagnóstico para um determinado valor de probabilidade.

A figura 2 apresenta a interface da aplicação desenvolvida.

\footnotetext{
${ }^{1}$ http://www.xspirit.de
} 


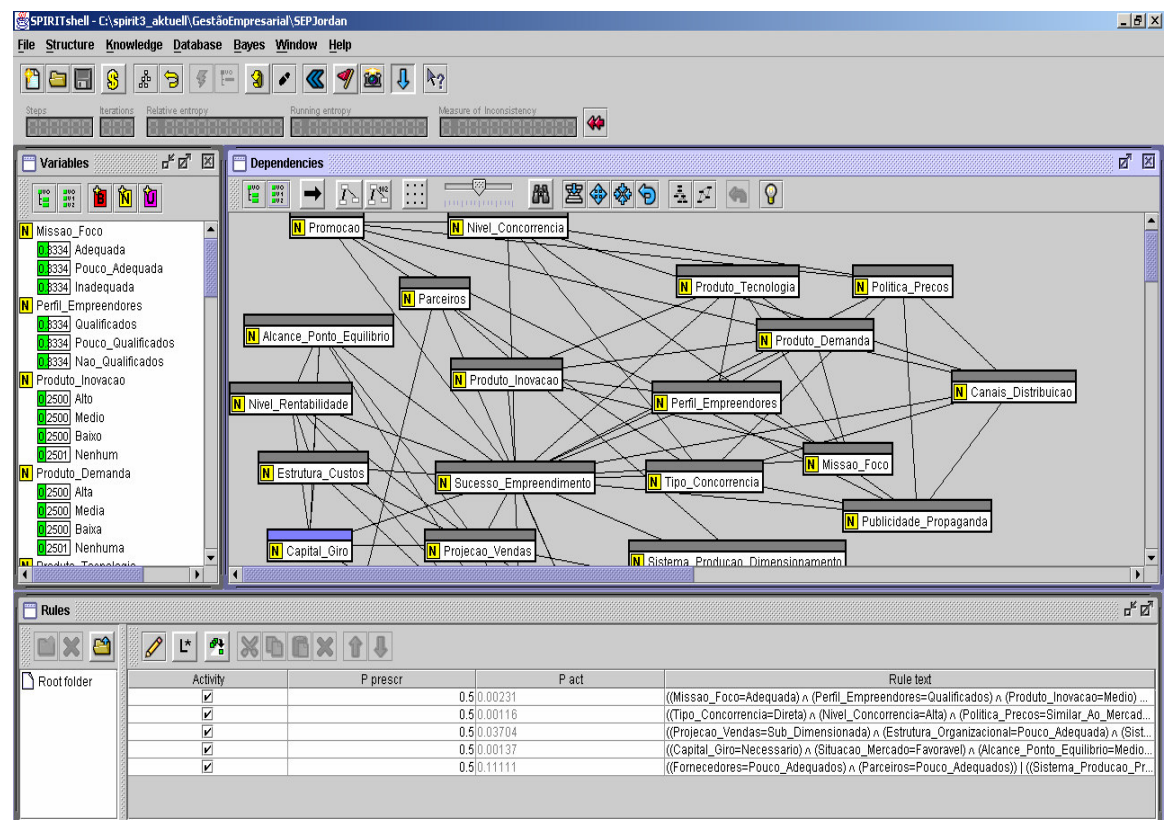

Figura 2 - Interface da shell utilizada.

\section{SHELL SPIRIT}

Sistemas baseados em conhecimento começaram a ser utilizados na década de 70 , entretanto eram baseados em regras puramente determinísticas. Atualmente é possível encontrar ferramentas que possibilitam o manuseio de regras que levem em conta incertezas e a subjetividade, SPIRIT (Symmetrical Probabilistic Intensional Reasoning in Inference Networks in Transition) é um software que possui essas características.

Segundo informações da página do projeto, trata-se de um dos mais sofisticados sistemas de shell utilizados para produção de sistemas inteligentes já construidos. Com ele, é possível utilizar estimativas subjetivas de probabilidades em um determinado domínio de conhecimento assim como dados estatísticos para construir uma base de conhecimento. Resumindo, SPIRIT é um tipo de sistema inteligente probabilístico, pois utiliza uma base de conhecimento baseada na distribuição da probabilidade.

O shell foi desenvolvido e é mantido por um grupo de pesquisadores da Universidade de Hagen na Alemanhã tendo como coordenador do projeto o prof. Dr. Wihelm Röder. Sua versão atual é a 3.1 e foi programada utilizando-se a linguagem java, portanto pode ser utilizada em diversos sistemas operacionais. Tendo sido testada em diversas versões do sistema operacional windows e também em ambientes linux e unix. Sendo distribuído através da GNU Public License ele pode ser baixado gratuitamente na internet.
Um software desse tipo pode ser utilizado em diversas aplicações. O SPIRIT já foi utilizado para construção de software de diferentes áreas como no software caso do ANALYST que é um sistema de suporte a decisão para liberação de empréstimos financeiros e também o software BANGANG onde uma parte da medicina chinesa foi modelada. Pode-se ainda citar o jogo Virtual 3 onde o usuário pode utilizar o SPIRIT para tomar as melhores decisões para atingir as meta do jogo.

\section{Conclusões}

O desenvolvimento de planos de negócio, como forma principal para 0 ingresso em incubadoras de empresa de tecnologia, é um processo muitas vezes lento e custoso, caracterizado por um alto esforço na realização de pesquisas, na definição do conceito do produto, na formatação de estratégicas comerciais e na estruturação financeira do projeto da empresa.

$\mathrm{O}$ trabalho intrinsicamente subjetivo $\mathrm{e}$ pouco metodológico de análise de planos de negócio, como forma de julgar novos projetos de empresas de base tecnológica, tem como resultado para as incubadoras um alto risco de não apoiar projetos com real potencial de desenvolvimento, desestimulando o empreendedorismo e prejudicando a economia local. O emprego dos sistemas especialistas probabilísticos, neste sentido corresponde a uma alternativa interessante para a minimização desta problemática, através da formalização do 
conhecimento tácito e procedural, empregado pelos consultores especializados, na análise dos planos. Visando o aprimoramento desta iniciativa, sugere-se a seleção de consultores especializados visando à formação de um time destinado adequação dos atributos e a formulação de novas regras de produção agregando na base de conhecimentos do sistema especialista a experiência dos diferentes profissionais. Destaca-se neste sentido a usabilidade da interface SPIRIT para a manipulação dos atributos, probabilidades e regras de compõem o sistema.

Ressalta-se também a possibilidade da experimentação de outras técnicas de inteligência artificial tais como redes neuronais e raciocínio baseado em casos, como forma de otimização da solução.

\section{Referências}

[1] Durkin, John. "Expert Systems. Design \& Development." Printice Hall, 1994.

[2] Kopittke, Bruno. "SPIRIT a new tecnology for expert systems." Disponível em: http://www.xspirit.de.
[3] Mattos, Merisandra Côrtes de. Sistema Especialista Probabilístico para Prognóstico de Doenças Bucais - PROBUCAL. Disponível em: http://www.avesta.com.br/anais/dados/trabalhos/3 61.pdf

[4] Nassar, Silvia Modesto. "Tratamento de Incertezas: Sistemas Especialistas Probabilísticos". Material utilizado em disciplina do Curso de Pós-Graduação em Ciência da Computação da UFSC. Site em http://inf.ufsc.br/silvia. 2003.

[5] Pavani, C. Plano de Negócios: "Planejando o sucesso de seu empreendimento". Rio de Janeiro: Lexikon editorial, 1997.

[6] Pizo, Carlos Antonio. Planos de negócios para empresas virtuais: uma análise do uso especificidades. 2002. 125f.. Dissertação (Mestrado em Engenharia de Produção) Programa de Pós-graduação em Engenharia de Produção, UFSC, Florianópolis.

[7] Rabuske, Renato Antônio. "Inteligência Artificial'. Florianópolis : Editora da UFSC, 1995.

[8] Ramos, Ronaldo F. Sistemas Especialistas "Uma Abordagen Baseada em Objetos com Prototipagem de um Selecionador de Processos de Soldagem", Dissertação de Mestrado. Universidade Federal de Santa Catarina, 1995. 